\title{
Electromagnetic radiation in heavy oil recovery process. Assessing a numerical model to predict the oil mobility
}

\author{
V. Pilipovik ${ }^{1}$ C. Riverol $^{2}$
}

Received: 19 August 2014/ Accepted: 17 May 2015/Published online: 29 May 2015

(C) The Author(s) 2015. This article is published with open access at Springerlink.com

\begin{abstract}
New recovery technologies are having an impact on heavy oil production which makes many marginal projects profitable. Before applying any technology or investing any capital in that, it is very important to have a model that can predict the results or oil recovery ratios. This paper deals with a model for predicting the flow in porous media of heavy oil using one of these emerging technologies (electromagnetic field) for heavy oil recovery. Some experiments were performed using different scenarios and thus evaluate the accuracy of the model developed in this article. It was found that the time and the frequency of the waves are key factors in promoting oil production. Also the article presents preliminary results of the model which will be useful for selecting the optimal frequency and time to stimulate heavy oil production to industrial scale.
\end{abstract}

Keywords Heavy oil · Model · Oil recovery . Electromagnetic waves $\cdot$ Simulation

\section{Introduction}

Due to the declining reserves facing conventional crude there has been a call for improving techniques for delivering a rich supply of heavy oil that is available worldwide (Hascakir et al. 2008). Electromagnetic waves can be considered as alternative for heavy oil and bitumen recovery. This method offers the following advantages:

C. Riverol

Carmen.riverol@sta.uwi.edu

JC. Engineering, Av. Libertador, Caracas, Venezuela

2 Department of Chemical Engineering, University of the West Indies, Trinidad, Republic of Trinidad and Tobago a. Reduction in the use of chemicals such as acids and solvents.

b. More precise stimulation of the wellbore can be accomplished as it can be directed to any defined interval.

c. It can be carried out during production of the well.

Recovery rates are determined by the mobility of the crude within a formation (Kovaleva et al. 2011). While artificial and enhanced oil recovery techniques may help in this matter, it is important to test and simulate the process before a decision on enhanced recovery can be recommended. A model for interfacial area dynamics based on pore scale is necessary for the simulation of the multiphase porous media.

In the literature, some references can be found, however few of them display a model that can be used for predicting the behavior of the technique before it can be implemented (Hascakir et al. 2008; Kovaleva et al. 2011; Sahimi 1995; Haque 1999). The phenomenon is based on molecular structures of the oil and porosity of the soil basically. Oils in different deposits vary in chemical properties and characteristics of the soil. The estimation of the size of the pore channel is a crucial factor for estimating the production of oil and permeability (Kovaleva et al. 2011; Masliyah et al. 2008). The objective of this article is to create a model for predicting the dynamic conditions and production of the well under electromagnetic radiation. This model can allow the user to estimate the effects of the method on oil production and it can be used in field-scale reservoirs.

\section{Experimental setup and model}

Multiphase porous media systems are characterized by fluid-fluid interfaces that exist at pore scale (Dullen 1992) and (Sahimi 1995). Dynamic pore scale models track the 
position based on boundary conditions fluid and solid properties and geometric characteristics. Each fluid follows a volume conservation law, such that

$V_{i} \frac{\partial V_{s i}}{\partial t}+\sum_{j}^{N} Q_{i j}=0$

where $V$ is the volume of the pore body, $V_{\mathrm{s}}$ is the local saturation, $N$ is the number of the pore in the body $(31 \%$ approximately), and $Q$ the volumetric flow from pore $i$ to pore $j$. In order to calculate the volume average in saturation, a representative volume should be defined where the volumetric flow is related to pressures using Poiseuillés law

$Q_{i j}=\operatorname{Ch}\left(P_{i}-P_{j}\right)$,

where $\mathrm{Ch}$ is the hydraulic conductance and $P$ the pressure. Boundary pressure is fixed to $300 \mathrm{kPa}$. In all simulations, we assume that the contact angle is zero and the radii of the pore bodies and pore throats are generated using a cut-off log normal distribution using upper and lower radius values shown. Finally the volume average velocity is given by the sum of each interfacial velocity weighted by area of interface.

$v=\frac{1}{A} \sum_{i} a_{i} v_{i}$,

where $a$ is the interfacial area per volume and $A$ is the total interfacial area (Davletbaev et al. 2011). By capillary theory, the radius can be estimated according to

$r=\frac{-2 \sigma \cos \alpha}{p}$

where $p$ is pressure and $\sigma$ is the surface tension. The energy balance is not included because the system was set up at a constant temperature. Assuming symmetry in radial homogeneous conducting medium (Bientinesi et al. 2013; Santos et al. 2011). The radial pressure distribution will be calculated as follows

$\frac{\mathrm{d} \phi}{\mathrm{d} r}=-\left(\gamma+\frac{1}{r}\right) \varphi$

where $\gamma$ is the power absorption and depends on the type of material and $\varphi$ is the power per unit area $\left(\mathrm{W} / \mathrm{mm}^{2}\right)$

As electromagnetic energy is absorbed by porous media, the relationship between temperature and energy is

$\Phi / r=\rho c_{p} \mathrm{~d} T$

The methods of electromagnetic radiation could be considered a "cold production" (without heating) thus $\mathrm{d} T$ can assume constant at $5{ }^{\circ} \mathrm{C}$ maximum. This Eq. (6) allows us to predict the density according to the electromagnetic radiation applied to the system. The efficiency of the electromagnetic field at experimental scale does not depend on the polar components of the oil.

The experimental setup consists in a simple pressured cylindrical vessel $(100 \mathrm{~cm}$ diameter $)$ inside water bath (30 $\mathrm{cm}$ diameter and $150 \mathrm{~cm}$ long) to keep the temperature between 20 and $25^{\circ} \mathrm{C}$. The vessels were tested before for avoiding leaks. A wave generator was installed at the top of the cylinder and it can work at different frequencies. During sand oil filling, uniform distribution of the sands was achieved. An oil collector and a thermocouple were located at the bottom the cylinder. See Fig. 1 .

A number of the experiments were performed using different types of oil sands. The height of the sands, frequency, time of the application of the wave, and gravity were the variables used for evaluating the performance of the model. Many experiments were setup, however, in this

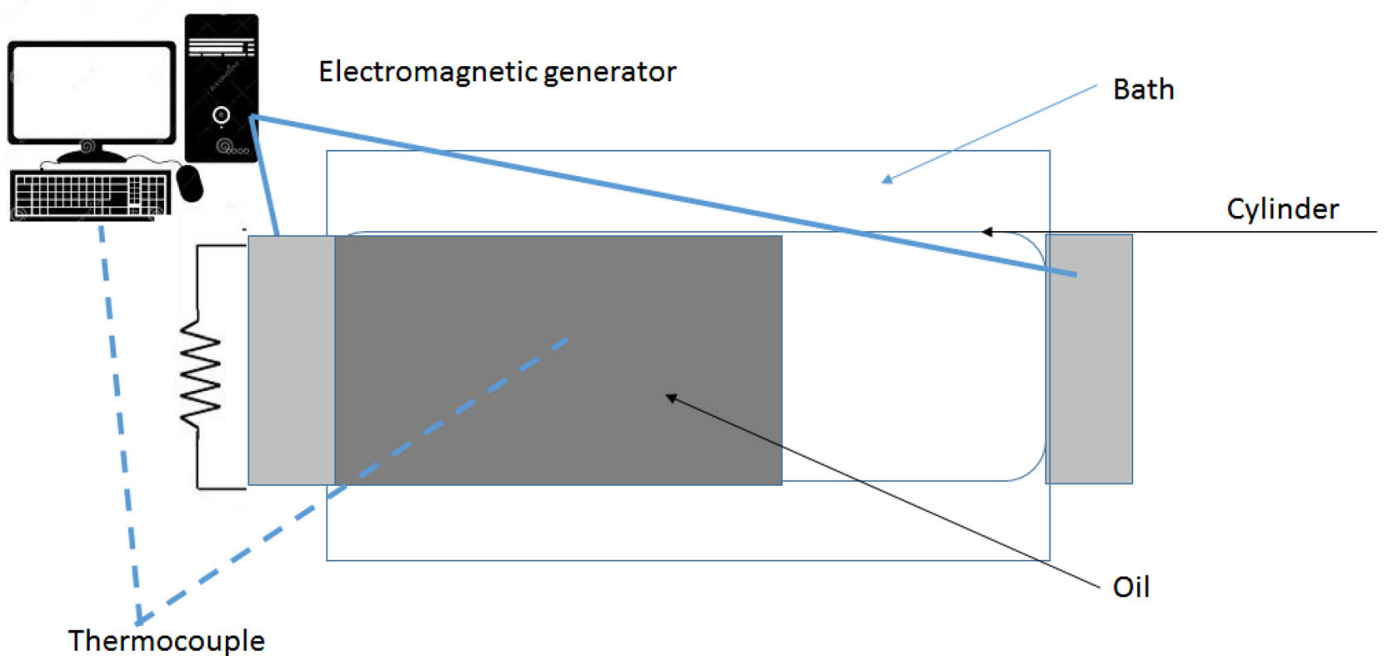

Fig. 1 Schematic representation of the experimental setup 
Table 1 Influence of the gravity at $50 \mathrm{~cm}$ and $250 \mathrm{MHz}$

\begin{tabular}{|c|c|c|}
\hline Gravity (API) & Velocity $(\mathrm{mm} / \mathrm{s})$ (average) & Velocity $(\mathrm{mm} / \mathrm{s})($ model $)$ \\
\hline 8 & 26 & 27.1 \\
\hline 9 & 30 & 31.3 \\
\hline 10 & 36 & 36.1 \\
\hline
\end{tabular}

Table 2 Influence of the gravity at $50 \mathrm{~cm}$ and $100 \mathrm{MHz}$

\begin{tabular}{lll}
\hline Gravity (API) & Velocity $(\mathrm{mm} / \mathrm{s})($ average) & Velocity $(\mathrm{mm} / \mathrm{s})$ (model) \\
\hline 8 & 27 & 27.1 \\
9 & 29 & 30.4 \\
10 & 36 & 35.6 \\
\hline
\end{tabular}

Table 3 Influence of the height at 9 API and $250 \mathrm{MHz}$

\begin{tabular}{lll}
\hline Height $(\mathrm{cm})$ & Velocity $(\mathrm{mm} / \mathrm{s})$ (average) & Velocity $(\mathrm{mm} / \mathrm{s})($ model $)$ \\
\hline 25 & 25 & 26.1 \\
35 & 31 & 30.4 \\
50 & 36 & 36.3 \\
60 & 44 & 44.1 \\
80 & 54 & 34.1 \\
\hline
\end{tabular}

Table 4 Influence of the time of application of the EM at $250 \mathrm{MHz}$

\begin{tabular}{lll}
\hline Time $(\mathrm{h})$ & Velocity $(\mathrm{mm} / \mathrm{s})$ (average) & Velocity $(\mathrm{mm} / \mathrm{s})(\mathrm{model})$ \\
\hline Oil sands at & 8 API and $50 \mathrm{~cm}$ height & \\
0 & 20 & 20.2 \\
8 & 35 & 37.2 \\
15 & 48 & 47.2 \\
25 & 56 & 55.5 \\
Oil sands at 9 API and $50 \mathrm{~cm}$ height & \\
0 & 20 & 20.2 \\
8 & 31 & 29.3 \\
15 & 46 & 47.8 \\
25 & 54 & 54.8 \\
Oil sands at & 10 API and $50 \mathrm{~cm}$ height \\
0 & 20 & 20.2 \\
8 & 29 & 32.1 \\
15 & 42 & 41.7 \\
25 & 51 & 51.5 \\
\hline
\end{tabular}

article, we include the more relevant results. All results are depicted in the Tables 1, 2, 3, 4, and 5. The maximum frequency used was $250 \mathrm{MHz}$ because the production reached a maximum when the frequency is equal to $250 \mathrm{MHz}$ which is independent of the density of the oil. Any frequency over that value does not increase the production significantly, see Tables 1 and 2.
Table 5 Influence of the frequency at $50 \mathrm{~cm}$ and 9 API

\begin{tabular}{lll}
\hline $\begin{array}{l}\text { Frequency } \\
(\mathrm{MHz})\end{array}$ & $\begin{array}{l}\text { Velocity }(\mathrm{m} / \mathrm{s}) \\
\text { (average) }\end{array}$ & $\begin{array}{l}\text { Velocity }(\mathrm{mm} / \mathrm{s}) \\
\text { (model) }\end{array}$ \\
\hline 80 & 34 & 34.2 \\
150 & 46 & 45.3 \\
250 & 54 & 54.1 \\
\hline
\end{tabular}

To assess the effect of the height in the production, the results are shown in the Table 3 . The results also reveal that the increase in the oil production was more pronounced at large height because it contains more oil.

All results were analyzed (included or not in this article), we found that the three key requirements in this method are: the exposed time to the wave, the frequency, and the gravity (API). These requirements allow to increase the production in the well significantly. Among different schemes it was observed that this technique never lets the well fall below the base oil production. This finding suggests that any wave frequency and or short-time application stimulates the oil production.

\section{Conclusion}

Overall, we believe that the model captures many of the essential features of flowing heavy oil and the effect of the electromagnetic field on the sands. The different variations of time and frequency should be considered depending on the reservoir and fluid properties. The results showed in this article were obtained to small scale, thus electrical conductivity should increase with increasing water saturation in big fields. We are currently working this model to real scale. We expect to continue to use the same framework presented herein to calculate the average velocity nevertheless, the electromagnetic field generator used at industrial scale is different from the generator used in the laboratory. Microwave energy was depicted to be effective in some applications; however, it is not used commercially at the present time.

Open Access This article is distributed under the terms of the Creative Commons Attribution 4.0 International License (http:// creativecommons.org/licenses/by/4.0/), which permits unrestricted use, distribution, and reproduction in any medium, provided you give appropriate credit to the original author(s) and the source, provide a link to the Creative Commons license, and indicate if changes were made.

\section{References}

Bientinesi M, Petarca L, Cerutti A, Bandinelli M, De Simoni M, Manotti M, Maddinelli G (2013) A ratio frequency/microwave 
heating method for thermal heavy oil recovery base don a novel tight-shell conceptual design. J Petrol Sci Eng 107:18-30

Davletbaev A, Kovaleva L, Babadagli T (2011) Mathematical modeling and field application of heavy oil recovery by radiofrequency electromagnetic stimulation. $J$ Petrol Sci Eng 78(3-4):646-653

Dullen F (1992) Porous media: fluid transport and pore structure, 2nd edn. Academic Press, San Diego

Haque K (1999) Microwave energy for mineral treatment processesa brief review. Int J Miner Process 57:1-24

Hascakir B, Babadagli T, Askin S (2008) Experimental and numerical modeling of heavy-oil recovery by electrical heating. In: Proceedings of the SPE, Paper 117669, Calgary, 20-23 Oct
Kovaleva L, Davletbaev A, Babadagli T, Stepanova Z (2011) Effects of electrical and radio-frequency electromagnetic heating on the mass-transfer process during miscible injection for heavy oil recovery. Energy Fuel 25:482-486

Masliyah J, Zhou Z, Xu Z, Czarnecki J, Hamza H (2008) Understanding water-based bitumen extraction from Athabasca oil sands. Can J Chem Eng 20:628-654

Sahimi M (1995) Flow and transport in porous media and fractured rock, 1st edn. Germany VHC, Weinheim

Santos MD, Neto AD, Mata W, Silva JP (2011) New antenna modelling using wavelets for heavy oil thermal recovering methods. J Pet Sci Eng 76(1-2):63-75 